\title{
Supporting Cells Contribute to Control of Hearing Sensitivity
}

\author{
Åke Flock, ${ }^{1}$ Britta Flock, ${ }^{1}$ Anders Fridberger, ${ }^{1}$ Eric Scarfone, ${ }^{2}$ and Mats Ulfendahl ${ }^{3}$ \\ ${ }^{1}$ Department of Physiology and Pharmacology, Karolinska Institutet, S-171 77 Stockholm, Sweden, 2Institut National de la \\ Santé et de la Recherche Médicale U432, Université Montpellier II, F-34095 Montpellier, France, and 'Ear, Nose, and \\ Throat Research Laboratory, King Gustaf V Research Institute, Karolinska Sjukhuset, S-171 76 Stockholm, Sweden
}

The mammalian hearing organ, the organ of Corti, was studied in an in vitro preparation of the guinea pig temporal bone. As in vivo, the hearing organ responded with an electrical potential, the cochlear microphonic potential, when stimulated with a test tone. After exposure to intense sound, the response to the test tone was reduced. The electrical response either recovered within 10-20 min or remained permanently reduced, thus corresponding to a temporary or sustained loss of sensitivity.

Using laser scanning confocal microscopy, stimulus-induced changes of the cellular structure of the hearing organ were simultaneously studied. The cells in the organ were labeled with two fluorescent probes, a membrane dye and a cytoplasm dye, showing enzymatic activity in living cells. Confocal microscopy images were collected and compared before and after intense sound exposure. The results were as follows. (1) The organ of Corti could be divided into two different structural entities in terms of their susceptibility to damage: an inner, structurally stable region comprised of the inner hair cell with its supporting cells and the inner and outer pillar cells; and an outer region that exhibited dynamic structural changes and consisted of the outer hair cells and the third Deiters' cell with its attached Hensen's cells. (2) Exposure to intense sound caused the Deiters' cells and Hensen's cells to move in toward the center of the cochlear turn. (3) This event coincided with a reduced sensitivity to the test tone (i.e., reduced cochlear microphonic potential). (4) The displacement and sensitivity loss could be reversible. It is concluded that these observations have relevance for understanding the mechanisms behind hearing loss after noise exposure and that the supporting cells take an active part in protection against trauma during high-intensity sound exposure.

Key words: hearing loss; inner ear; cochlea; hair cells; supporting cells; acoustic trauma
Overstimulation by intense sound gives rise to several structural and functional effects in the organ of Corti, the mammalian hearing organ. The structural effects include a shrinking of the tectorial membrane (Canlon, 1987), disruption of the tip-links of the stereocilia (Pickles et al., 1987), fracture of the actin core and bending of the stereocilia (Engström et al., 1983; Saunders et al., 1991), shortening and swelling of outer hair cell (OHC) bodies (Ulfendahl et al., 1993), contortion, blebbing, and degeneration of the cell body (Spoendlin, 1971), distension of the Deiters' cells (DCs) (Raphael et al., 1993), swelling of the afferent nerve endings (Eybalin, 1993), and degeneration of afferent neurons (Lim, 1976). The morphological signs of damage are usually accompanied by several pathophysiological consequences (for review, see Saunders et al., 1991; Borg et al., 1995). With few exceptions, the structural effects of noise trauma have been studied in material fixed at a certain point in a physiological experiment and processed for subsequent histological examination of sections from the embedded organ. We have recently developed a technique to perform laser scanning confocal microscopy

\footnotetext{
Received Nov. 16, 1998; revised March 4, 1999; accepted March 10, 1999.

This work was supported by the Swedish Medical Research Council Grant 02461, the Knut and Alice Wallenberg Foundation, the Foundation Tysta Skolan, the Swedish Association of Hard of Hearing, the Swedish Society for Medical Research, and funds from the Karolinska Institutet and Institut National de la Santé et de la Recherche Médicale. Parts of this project were performed within the framework of the European BIOMED and BIOTECHNOLOGY research programs (BMH1CT94-1793, BIO4-CT98-0408; coordinator Eric Scarfone). We thank Dr. J. D. Bruton for helpful comments and suggestions.

Correspondence should be addressed to Åke Flock, Department of Physiology and Pharmacology, Karolinska Institutet, S-171 77 Stockholm, Sweden.

Copyright (C) 1999 Society for Neuroscience $0270-6474 / 99 / 194498-10 \$ 05.00 / 0$
}

(LSCM) of the living organ of Corti, vitally stained by fluorescent probes (Flock et al., 1998a) in an in vitro preparation of the guinea pig temporal bone (Ulfendahl et al., 1989). This provides a possibility to observe all of the structures mentioned above, except the tip-links, and their response to acoustic stimulation, while at the same time recording electrophysiological events. Because the structure and function of the organ of Corti can be monitored for several hours (Ulfendahl and Flock, 1998), both immediate and long-term effects of acoustic overstimulation can be studied.

In a recent study, we combined electrophysiological recordings of cochlear potentials with simultaneous observations of organ structure and measurement of intracellular free calcium in the isolated temporal bone preparation (Fridberger et al., 1998). A new structural phenomenon was found to develop as a consequence of intense sound stimulation, consisting of a narrowing of the width, or "contraction," of the organ. In $\sim 50 \%$ of cases, the response was temporary and recovered, in part or completely, within 30-45 min. In the remaining cases, the displacement was sustained and did not reverse within the time of observation. In the present work, we have explored the cellular origin of this structural effect using confocal microscopy, in combination with electrophysiological measurements of cochlear potentials during acoustic stress. We suggest that the displacement is a protective response of the organ of Corti against injury.

\section{MATERIALS AND METHODS}

\section{The preparation}

The present studies on the hearing organ were performed using temporal bones from pigmented guinea pigs maintained in an experimental cham- 


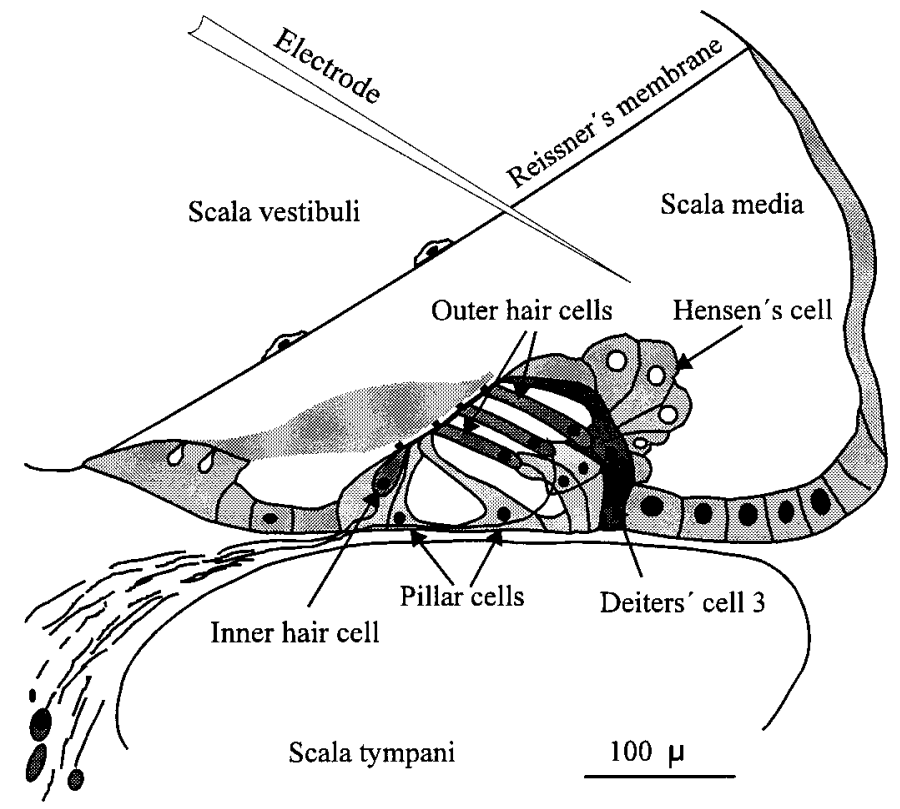

Figure 1. Schematic drawing of the cochlear partition showing its structural components and the position of the microelectrode.

ber filled with tissue culture medium (Ulfendahl et al., 1989). The animals were obtained from the Sahlin Animal Farm (Malmö, Sweden). The experiments were approved by the Ethics Committee of the Karolinska Institutet (permit no. N124/96), and all efforts were made to minimize animal suffering. The present report is based on 24 successful preparations. The temporal bone was mounted in a holder attached to the chamber, allowing rotations in two planes to provide proper alignment with respect to the optical axis of the microscope. A hole was drilled in the scala tympani of the basal turn of the cochlea and into which a thin plastic tube was inserted. The tubing was connected to a tank filled with oxygenated medium to which fluorescent probes could be added for direct application transport to the organ via the cochlear fluids. An opening was made in the bony shell covering the apex of the cochlea, allowing access to the organ for viewing and for microelectrode recordings (Fig. 1).

\section{Viewing}

Incident light and video-enhanced microscopy. Illuminating the preparation from the side by fiber optics, the organ was tilted as much as possible to obtain a close to radial section (Fig. 2) in the most basal part of the exposed cochlear turn, while still permitting the insertion of the microelectrode above the bony shell of the cochlea (Fig. 1). A $40 \times$ water immersion objective (NA, 0.75; working distance, $1.98 \mathrm{~mm}$; Zeiss, Oberkochen, Germany) was used for viewing. The light microscope image is formed by light diffracted from the specimen rather than by transmitted light, resulting in a Nomarski-like image. It was video enhanced by means of a television camera and an image processing system.

Confocal microscopy. Confocal microscopy was performed using an Medical Research Council 1024 system (Bio-Rad, Hertfordshire, UK) equipped with a krypton-argon laser providing using excitation in blue $(488 \mathrm{~nm})$ and appropriate emission filters for the two channels $(585 \mathrm{~nm}$ long-pass and $522 \mathrm{~nm}$ bandpass filters). All preparations were double labeled as described previously (Flock et al., 1997, 1998a) using two probes: RH414 (1-15 $\mu \mathrm{g} / \mathrm{ml}$; Molecular Probes, Eugene, OR), a styryl dye staining cell membranes, and calcein AM-ester $(4 \mu \mathrm{M} / \mathrm{ml})$, which is a substrate enzymatically cleaved in the cytoplasm in living cells to form a membrane-impermeant green fluorescent product. Calcein was added to the perfusion tank as soon as the perfusion started, whereas the more rapidly staining styryl dye was added $\sim 30$ min later. LSCM images were obtained in the $x-y$ plane with the organ tilted with respect to the focal plane of the microscope so that the resulting optical section had an orientation with respect to the organ, as illustrated in Figure 2. During an experiment, LSCM images were collected every 5-10 min to obtain a stack of 11 optical sections, each separated by $2.5 \mu \mathrm{m}$. The stack thus covered a $27.5 \mu \mathrm{m}$ depth of tissue, corresponding to approximately four

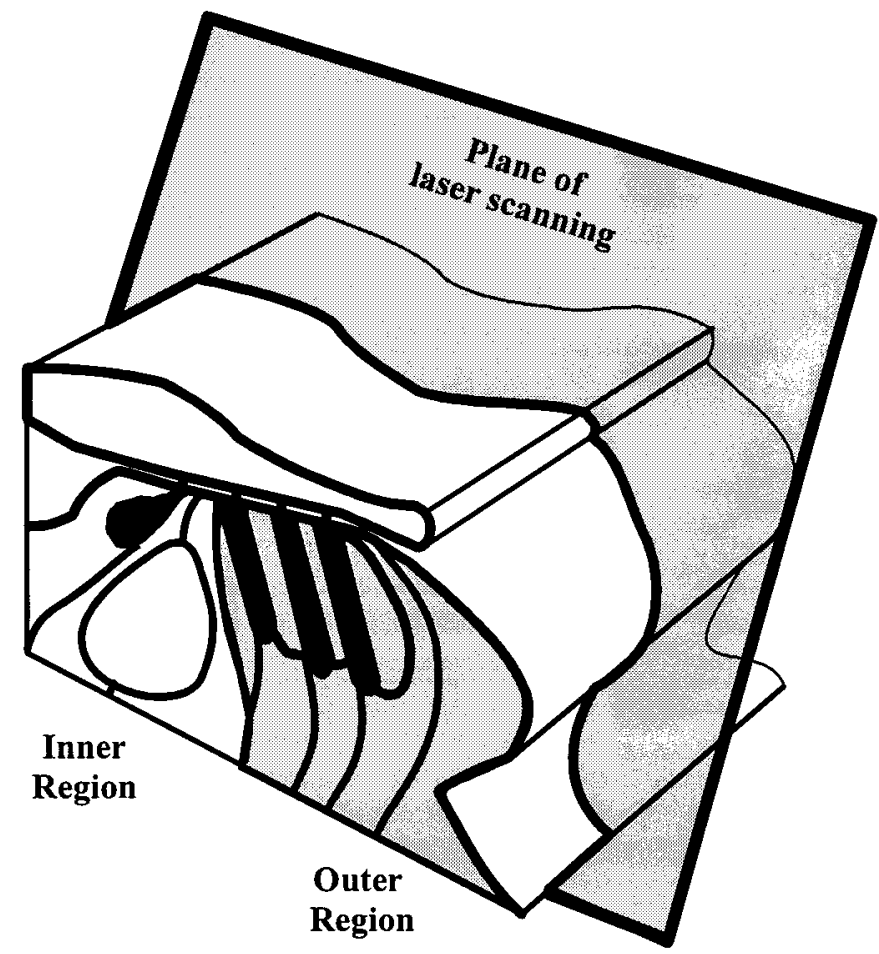

Figure 2. Schematic drawing illustrating how the preparation was tilted with respect to the plane of confocal laser scanning to obtain LSCM images, showing nearly radial sections of the organ of Corti (compare Figs. 1, 5).

to five OHCs and four inner hair cells (IHC) along the spiral dimension of the organ. In a typical experiment, nine stacks would be collected, three before the first exposure, three after the first exposure, and three after the second exposure. Within the stacks, corresponding sections were selected and compared. Quantitative measurements of motion were measured using cellular structures that could be followed throughout the stack. Usually, this was a lipid droplet in a Hensen's cell $(\mathrm{HeC})$ because its center is well defined.

Light microscopy. For light microscopy, glutaraldehyde was added to the perfusion tank to make a final concentration of $2.5 \%$. The cochlear microphonic potential $(\mathrm{CM})$ was observed until it vanished, and the preparation was transferred to fixative. For examination of the extent of cell damage, the apical cochlear turn was excised, mounted flat, and observed using differential interference contrast microscopy.

\section{Acoustic stimulation}

Sound delivery. The external auditory meatus of the temporal bone was press-fitted against a rubber gasket coupled to a loudspeaker via a plastic connector. A probe microphone was inserted to measure the sound pressure close to the external auditory meatus. All sound pressure values are given as decibels relative to $20 \mu \mathrm{Pa}$.

The sound stimulus. The sound was presented as test tones generated by a custom-made computer program and a waveform generator. Because immersing the middle ear cavity in tissue culture medium restrains the motion of the ear drum, the sound reaching the cochlea is reduced by $\sim 30 \mathrm{~dB}$ (Brundin et al., 1992). Sound pressure level (SPL) is therefore given as the value measured by the probe microphone minus $30 \mathrm{~dB}$. The test tone was presented in several ways: (1) as $140 \mathrm{~Hz}$ tone at $50 \mathrm{~dB}$ with a duration of $300 \mathrm{msec}$ and a repetition rate of $300 \mathrm{msec}$; (2) as a frequency sweep from 60 to $500 \mathrm{~Hz}$ at a constant SPL, generating iso-intensity curves (in the text, these are called tuning curves); and (3) as a frequency sweep in which, for each frequency, the SPL measured by the probe microphone at the external auditory meatus was adjusted to produce a $\mathrm{CM}$ held at a constant preset amplitude, generating iso-response curves (compensated for frequency-dependent distortion in the acoustic system to a limit determined by a preset tolerance level, usually $3 \mathrm{~dB}$ ).

Sound-exposure. By this term, we refer to a high-intensity pure tone at $140 \mathrm{~Hz}$. This was delivered as tone bursts as specified above. The 

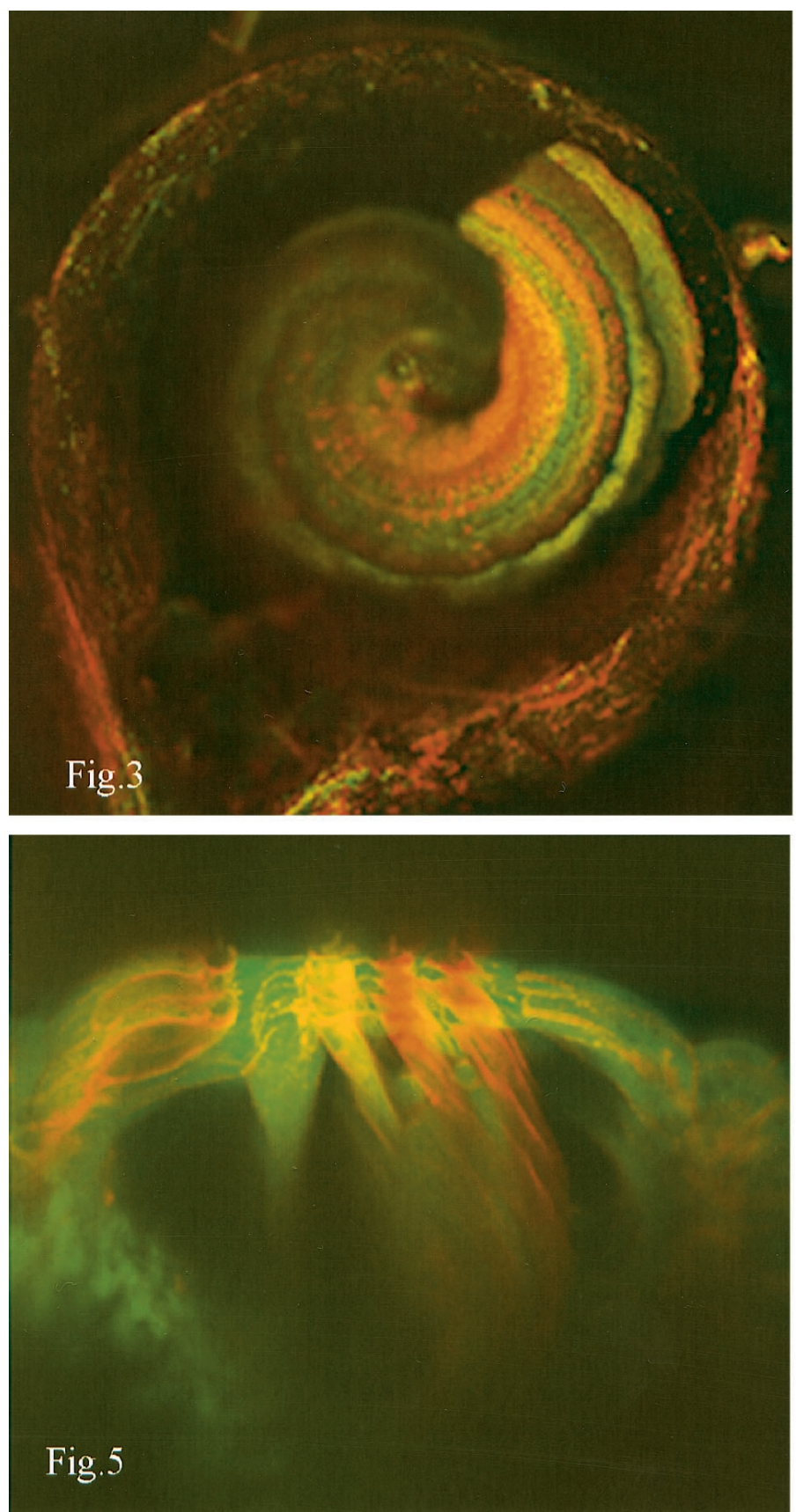

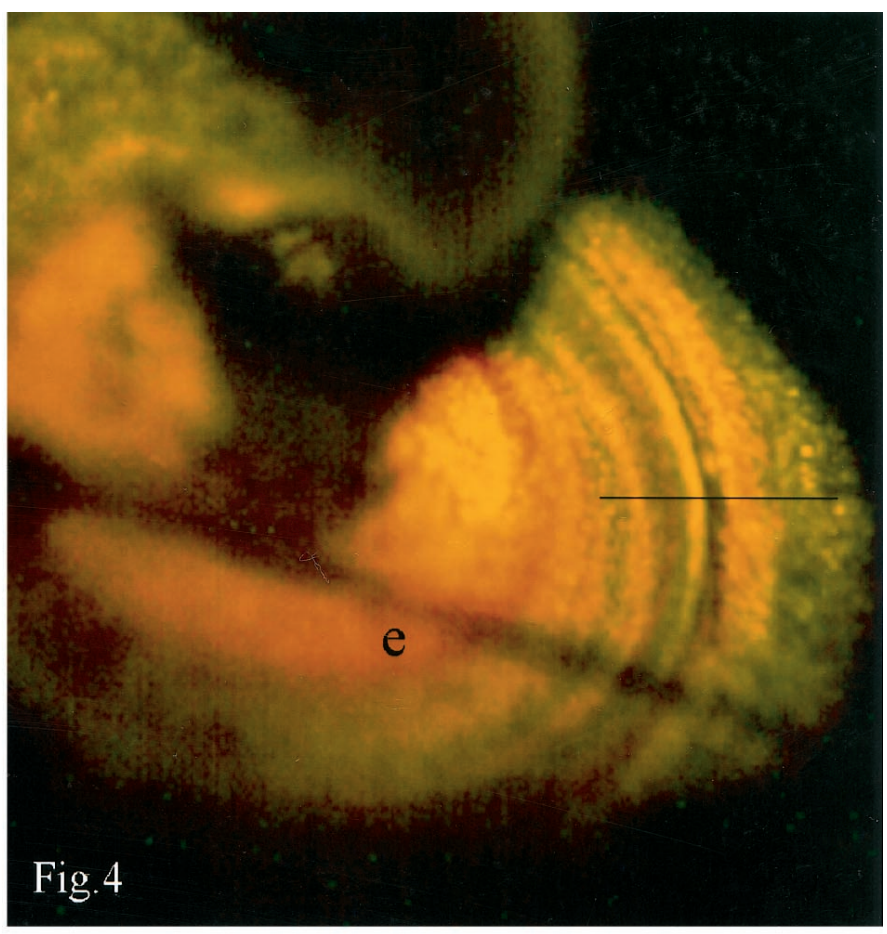

Figure 3. Low-power LSCM image of the apex of the cochlea with the bony shell removed to expose the organ of Corti. The image is an extended depth projection of 11 individual confocal sections acquired 10 $\mu \mathrm{m}$ apart. Concentric rings of different colors (red, green, yellow) correspond to specific cellular staining of the organ of Corti in which structures were stained by the two fluorescent dyes with varying intensity. The even red band is comprised of the OHCs, outside is an irregular red band of $\mathrm{HeCs}$, and inside is a green band of pillar cells. The inner red ring is made up by nerve fibers of the auditory nerve. Magnification, $52 \times$.

Figure 4. A preparation in which the opened cochlea is tilted to provide an appropriate angle (compare with Fig. 2) for acquiring LSCM images. The black line indicates the position of the plane of laser scanning through the organ of Corti, as seen in Figures 2, 5 and 6, 8. The oblique dark band is the electrode $(e)$ used for recording the cochlear microphonic potential. Magnification, $118 \times$.

Figure 5. Extended focal depth reconstruction of the organ of Corti acquired at a position corresponding to the line in Figure 4. Doublestaining with membrane dye (RH 414, red) and cytoplasmic enzyme dye (calcein, green). The same organ is seen with the two dyes separated in Figure 8 , and its electrophysiological response to sound exposure is shown in Figure 12. Magnification, 345×.

\section{RESULTS}

\section{Internal organ structure}

\section{Imaging and organ reconstruction}

A general schematic view of the scala media and the organ of Corti is shown in Figure 1, which also illustrates the location of the electrode recording the CM. Low-magnification $(4 \times$ objective) views of the cochlea with the apex opened are presented in Figures 3 and 4. In Figure 3, the cochlea is oriented with the apex facing straight up, and the organ of Corti is seen as a band spiralling down counterclockwise and disappearing beneath the bony overhang. In Figure 4, the cochlea is tilted to give a plane of laser beam scanning close to that illustrated schematically in Figure 2. The electrode, seen as an oblique dark band, was placed 

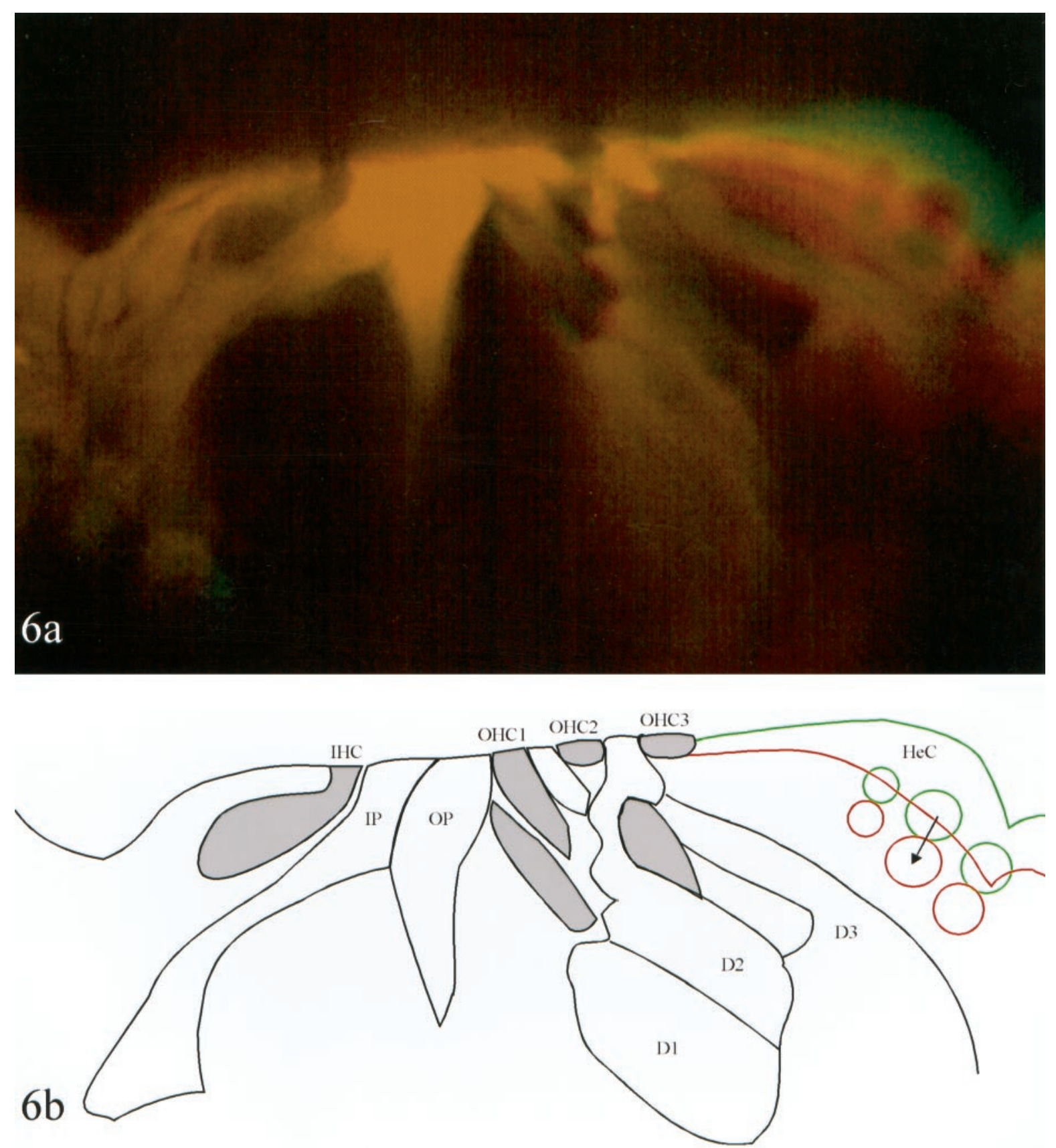

Figure 6. a, Sound-induced displacement of DC and HeCs. Two superimposed sections acquired before ( green) and after (red) sound exposure. Where the two sections match, the image is yellow. Magnification, $355 \times$. b, Drawing of cell profiles in Figure 6a. Sensory cells are shaded. The colored region to the right illustrates the motion as displayed by the red and green profiles. The arrow indicates the direction of displacement during sound exposure. $O H C 1, O H C 2, O H C 3$, Outer hair cells 1, 2, and 3, respectively; $I P$, inner pillar cell; $O P$, outer pillar cell; $D 1, D 2, D 3$, Deiters' cells 1, 2, and 3, respectively.

so as not to obscure the view of the region of the organ where the images were acquired.

A high-magnification $(40 \times$ objective) view of the organ of Corti is shown in Figure 5. It was obtained at the region indicated by the thin black line in Figure 4. It is composed from two stacks of 11 images, one for each of the dyes, the membrane dye RH414 (red) and the cytoplasmic enzyme dye calcein (green). The figure shows the sections superimposed, providing an extended depth projection. The images of the two dyes were acquired simultaneously and merged in the projection. The two dyes are presented separately (see Fig. $8 a-d$ ). Each stack provided a volume of reconstructed tissue approximately five $\mathrm{OHCs}$ deep, which is sufficient to allow for a slight drift in focus of the preparation over time and for changes in focus caused by the displacement of the organ after noise exposure.

Dynamic changes in organ structure after noise exposure: displacement of the Deiters' and Hensen's cell complex

The main purpose of the structural investigation was to search for the cellular origin of the decreased radial width of the organ of Corti seen in previous work from our laboratory (Fridberger et al., 1998). Analysis of images was now made on LSCM image stacks to look for structural changes inside the organ after sound exposure. The corresponding images before and after noise ex- 

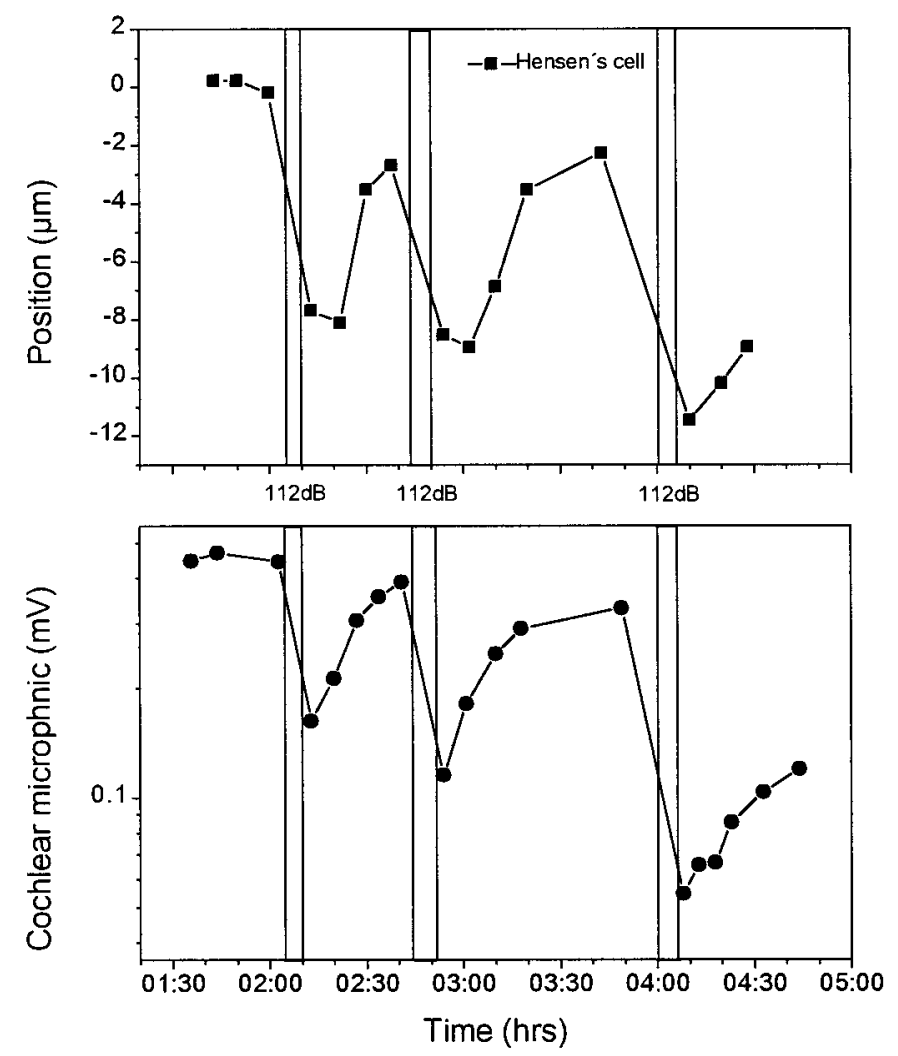

Figure 7. Temporary structural displacement and sensitivity loss. The top shows the position of a lipid droplet in a $\mathrm{HeC}$. A negative value corresponds to motion in the direction of the arrow in Figure 6b. After each of the three sound exposures, indicated by the bars, the structure had moved in this direction. During a period of recovery, the lipid droplet returned back toward its original position. The bottom shows that a reversible loss of sensitivity of the cochlear microphonic potential occurred in parallel.

posure were centered around the heads of the pillar cells, and a movie was made flip-flopping between the two. The key result is illustrated in Figure $6 a$ in which the LSCM image before noise is color coded in green, and the corresponding section after noise is coded in red. Figure $6 b$ is a camera lucida drawing to help identify the structures seen in the image. Where the two sections completely overlap, the image is yellow, and where structures had moved with respect to each other, the green-red areas separate. The two superimposed sections show that, after sound exposure, the HeCs had moved in a direction toward the tunnel of Corti. Most of the movement generally occurred around a hinge point between the third row DC (DC3) and the third row OHC around which the outer part rotates. This motion was usually accompanied by a tilting of the reticular lamina around a hinge point located at the first row of OHCs. Three lipid droplets are seen inside an $\mathrm{HeC}$. In the inset, a white arrow indicates the center-tocenter distance of motion. This distance is given as position in Figure 7 in which a negative value corresponds to a motion in the direction of the arrow.

The displacement could be reversed, in which case, no residual structural alterations remained in the organ. A residual structural effect was generally seen after exposure at an SPL of $106 \mathrm{~dB}$ or higher during the time limit of our experiments. In an unexposed preparation that had stable electrophysiological responses to the test tone, no relative motion between cells within the organ of Corti could be detected.

\section{Effects of sound exposure on general cellular structure}

The OHCs could swell and contract so that their bases with their nerve endings and the cups of the DCs are pulled up. OHCs shortened irreversibly in 11 preparations. This was accompanied by a sustained loss of CM. OHCs shortened reversibly in three preparations, and in three they elongated. The angle of the OHCs, with slender bodies, could change, their base swinging toward the tunnel of Corti. The package of OHCs plus DCs could swell symmetrically, row three pushing in the outward direction, row one pushing inward. Swollen phalangeal processes of DCs could then often be seen between the hair cells. In these cases, DC3 and HeCs could be seen to shift out toward the stria vascularis. Blebs could be seen to develop at the apical surface of the OHCs, and they would lose their enzyme activity, seen as a decease in the calcein stain. In general, rows three and two were more susceptible to damage than row one. In a number of preparations, the IHC performed a slight position shift or narrowing after sound exposure, moving its base toward the tunnel of Corti. The pillar cells are most stable and were never seen to buckle.

In Figure 8, the hearing organ presented in Figure 5 is seen with the two dyes separated (before and after sound exposure). The membrane dye (Fig. 8a) showed straight, smooth OHCs before sound exposure, as well as distinct staining of IHCs and their innervating nerve endings, and cell walls of pillar cells, DCs, and $\mathrm{HeCs}$. Intracellular membrane-bound organelles were stained to some extent. The calcein dye (Fig. 8c) staining (acquired simultaneously) showed the cytoplasm of both sensory and supporting cells. The pillar cells, $\mathrm{HeCs}$, and cells forming the bottom of the tunnel of Corti were vividly stained, whereas staining of IHCs and second and third row OHCs was less intense. The deeper aspects of the DCs were only faintly seen.

The preparation shown in Figure 8 was exposed to four incidents of sound exposure. The first three did not affect the hearing organ, whereas the fourth caused drastic structural changes (Fig. $8 b, d)$, which were also accompanied by a reduction of the CM (see Fig. 12). In Figure 8b, swollen and contracted OHCs can be seen after sound exposure. In Figure $8 d$, a loss of calcein staining is seen, especially in the first row of OHCs, and a tilt of these cells toward the tunnel of Corti is seen. The inner region around the IHC, pillar cells, and supporting cells seemed intact.

In summary, the structural investigation showed that the organ of Corti displays two structural regions illustrated schematically in Figure 2: (1) an inner region, which is the most structurally stable part consisting of the IHC, inner and outer pillar heads, basilar membrane at the base of the inner pillar, and nerve fibers in the habenula region; and (2) an outer less stable region comprised of the OHCs and the third row of DCs plus the HeCs.

\section{Distribution of structural damage}

To determine to what extent the region of observation with the confocal microscope matched the region of damage, five preparations were fixed for light microscopic observation of the excised uppermost turn of the cochlea. We wanted to ascertain whether the damage was limited to the region of illumination with laser light, in which case we would be dealing with irradiation damage, or whether the region of damage was related to the sound exposure.

Figure 9 shows the preparation from where Figure 5 and 8 were obtained. The preparation was fixed $\sim 10$ min after the images shown in Figure 8, $b$ and $d$, were obtained. It illustrates at the light microscopic level the extent of the region of noise damage in the preparation. Figure $9 a$ illustrates schematically where along the 

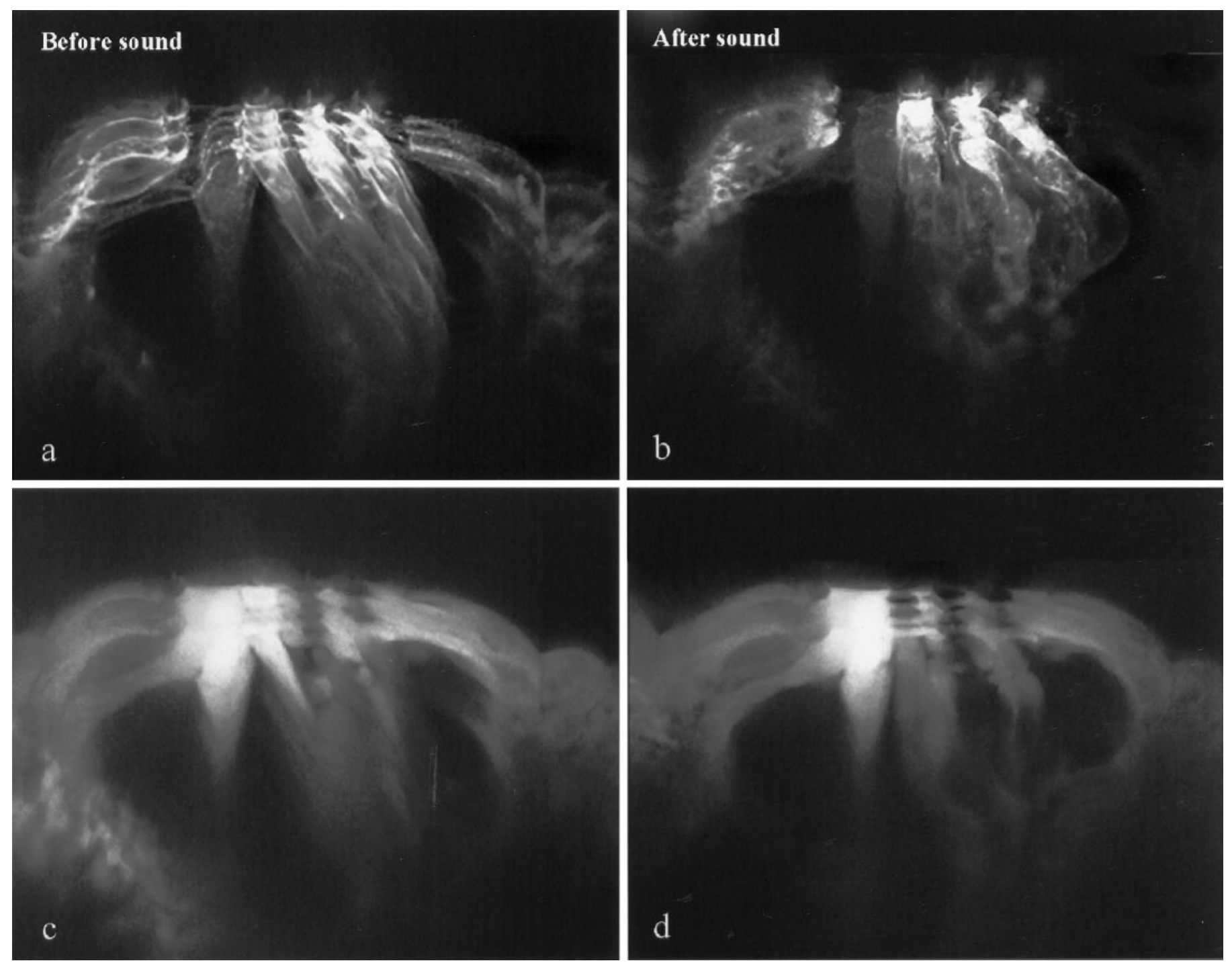

Figure 8. Structural effects of sound exposure. Images obtained before and after sound exposure for the membrane $(a, b)$ and the enzyme $(c, d)$ dyes. The reconstruction is the same as seen in Figure 5, but here the two dyes (recorded in separate channels) are separated. The physiological effect on this organ is illustrated in Figure 12. At the end of the experiment, the organ was fixed and examined using light microscopy, as shown in Figure 9. Magnification, $390 \times$.

cochlea the different areas were located, $\sim 18-19 \mathrm{~mm}$ from the base. Figure $9 b$ is a low-power view showing the regions where images $c-f$ were obtained. Figure $9, c$ and $d$, was acquired at two different focal planes in the region (region 1) where the LSCM images shown in Figures 5 and 8 were collected. They reveal damage to second and third row OHCs $(d)$, whereas the first row OHC bodies have the glistening appearance characteristic of healthy cells $(c)$. In Figure $9 e$, from region 2 at the more basal, high-frequency side of the turn, more extensive damage is seen. The cell bodies of the second and third row OHCs were swollen, and the first row was deranged and had lost its glistening appearance. Figure $9 f$ is from region 3 further toward the basal portion of the accessible part of the coil and shows undamaged long slender $\mathrm{OHC}$ walls. Throughout the affected region, the sensory hair bundles appeared surprisingly regular and undisturbed. These results show that the area of damage is not limited to the area of laser illumination but is frequency-dependent, engaging not only the region under observation but also more severely an area on the high-frequency side of this site. Because of the limited time of observation, we cannot judge to what extent this damage was reversible.

\section{Electrophysiological measurements}

When the microelectrode penetrated the Reissner's membrane into the scala media (Fig. 1), a CM was recorded in response to the test tone. Sweeping the frequency of the stimulating sound from 60 to $500 \mathrm{~Hz}$ changed the amplitude of the CM, showing a maximum at $145 \mathrm{~Hz}(\mathrm{SD}, \pm 9.4 ; n=21)$. At the best frequency, the amplitude of the CM was $0.22 \mathrm{mV}(\mathrm{SD}, \pm 0.15 \mathrm{mV} ; n=21)$ in response to the applied test tone, which had an intensity of 49 $\mathrm{dB}$ SPL (SD, $\pm 7.3 ; n=19)$. At higher stimulus intensities, the $\mathrm{CM}$ became biased toward the positive direction, forming a summating potential. In the initial phase of an experiment, the $\mathrm{CM}$ and frequency responses were repeatedly recorded to check the stability of the preparation. Unless the organ was soundexposed, the response was stable for $\sim 4 \mathrm{hr}$, although a gradual decline sometimes was seen.

Figure 10 shows iso-response curves obtained before and after 

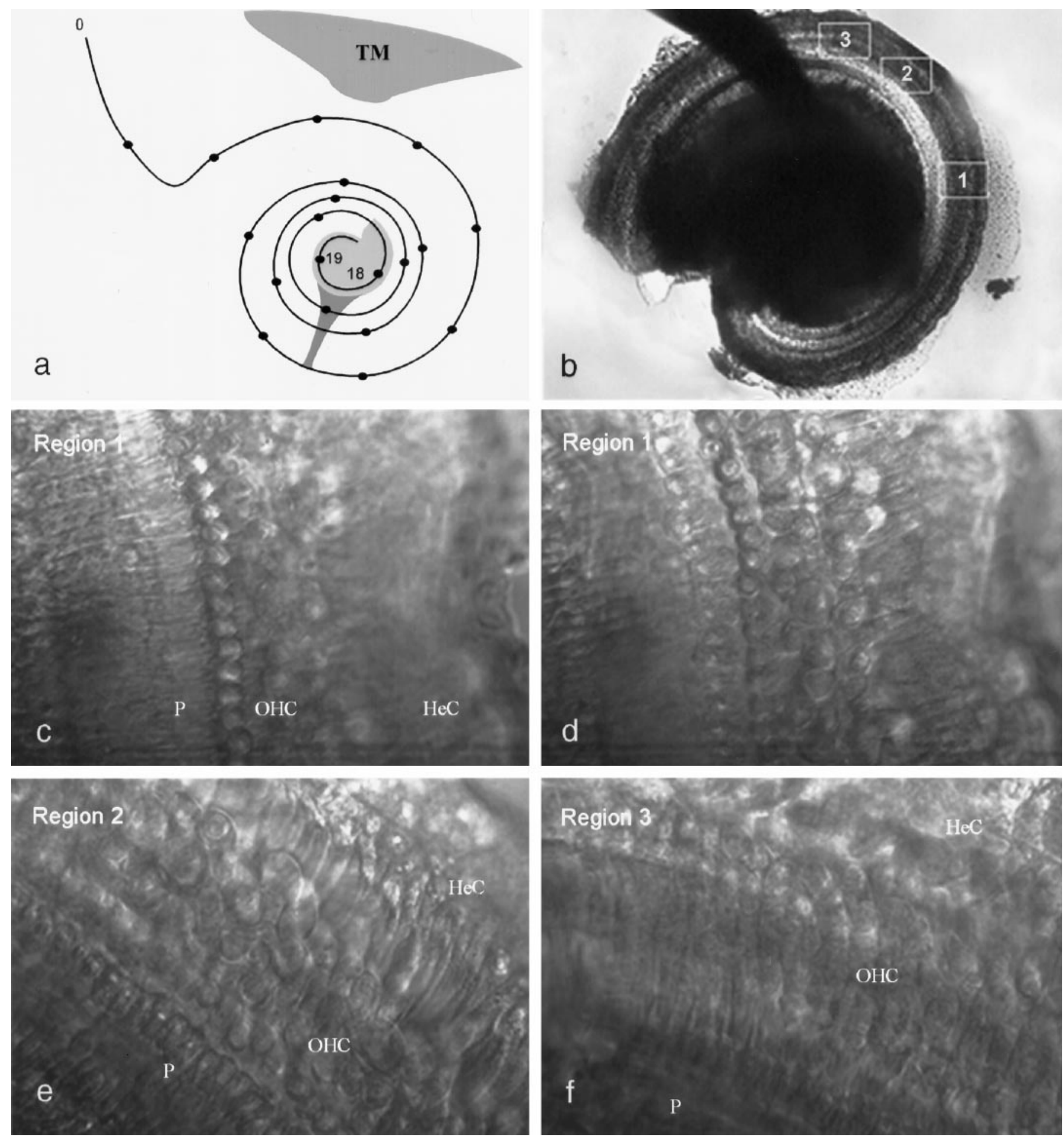

Figure 9. Montage demonstrating the distribution of structural damage caused by noise exposure. The preparation is the same as that presented in Figures 5, 8, and 12. $a$, Schematic drawing of the coils of the guinea pig cochlea with millimeter distances from the base (0) marked with points. The uppermost coil of the cochlea, the gray shaded area in the $18-19 \mathrm{~mm}$ region, is available for observation during an experiment in the confocal microscope. $b$, The organ was fixed, and the coil corresponding to the 18-19 mm shaded region shown in $a$ was excised and examined with Nomarski interference contrast microscopy (compare $c-f$ ). LSCM images were acquired in region 1, which is the same region from where the reconstructions shown in Figures 5 and 8 were obtained. The region is seen at two different focal planes in $c$ and $d$. Swollen OHCs of the second and third row are seen in $d . e$, The number of damaged cells was larger in region 2, which was situated on the high-frequency side of the point of observation. $f$, Region 3 was undamaged. TM, Tympanic membrane; $P$, pillar cell. Magnification: $b, 47 \times ; c-f, 423 \times$. 


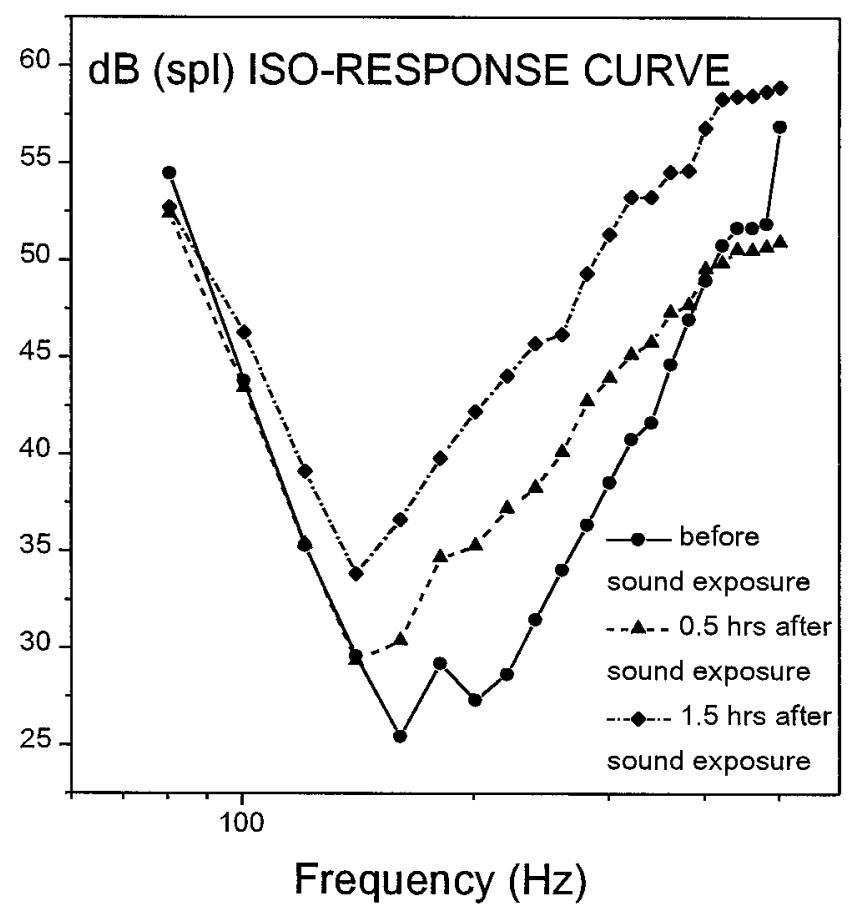

Figure 10. Iso-response curves for the cochlear microphonic potential (see Material and Methods) show loss of sensitivity in the high-frequency region and a shift of best frequency toward lower frequency after sound exposure.

two sound exposure sessions. The level of the test tone, measured at the external auditory meatus, needed to produce a $\mathrm{CM}$ of 2.5 $\mu \mathrm{V}$, is plotted as a function of stimulus frequency. Before sound exposure, the electrical response expressed a best frequency of 160-200 Hz. After the first sound exposure, there was a loss of sensitivity on the high-frequency side and a downward shift of best frequency to $140 \mathrm{~Hz}$. The second overexposure produced a further loss of sensitivity. The sensitivity loss was most pronounced on the high-frequency side, thus matching the distribution of structural damage shown in Figure 9.

When the intensity of the potentially traumatic sound exposure was increased step by step, the CM increased, saturated, and then diminished. It then stabilized at a reduced value. This sound exposure was maintained for $5 \mathrm{~min}$. When the sound exposure was terminated, either the CM returned to its original amplitude immediately, giving tuning curves identical to those obtained before, or it recovered gradually (Fig. 7). The recovery could be complete (Fig. 7, first exposure) or partial, leaving a residually reduced CM (see Fig. 12). The peak of the tuning curve was reduced in amplitude and shifted toward a lower frequency.

\section{Displacement and sensitivity loss}

In Figures 7 and 11, the position of the $\mathrm{HeC}$ and the amplitude of the $\mathrm{CM}$ are plotted against time. After an initial control period, the organ represented by Figure 7 was stimulated in three sessions by tone bursts at $140 \mathrm{~Hz}$ with an intensity of $112 \mathrm{~dB}$ for $5 \mathrm{~min}$. A structural change occurred during each sound exposure; the outer region moved toward the pillar cells, causing a change in position of $6.25 \mu \mathrm{m}(\mathrm{SD}, \pm 2.9 ; n=18)$. With time, the HeCs returned back toward their original location until the next exposure commenced. There was no other change in cell structure after the first and second sound exposure, but toward the end of the experi-
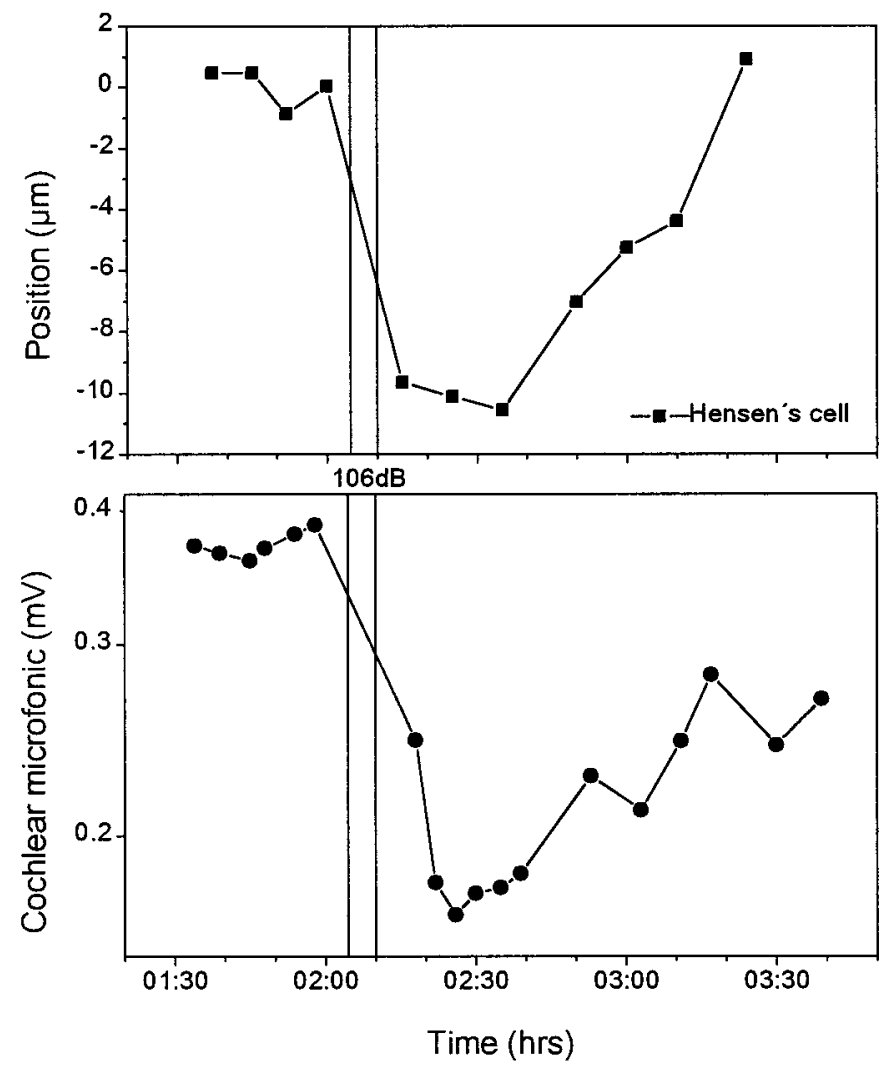

Figure 11. Long-term position shift and sensitivity loss. In this preparation, the structural recovery took $60 \mathrm{~min}$, whereas the microphonic potential recovered only partially during this time period.

ment, swelling of the third row of hair cells was seen. The CM followed the same pattern; after sound exposure, the sensitivity to the test tone was reduced. However, the loss was temporary in that the CM recovered toward its initial value. The recovery of the CM had a faster onset than that of the relaxation. This type of response was observed in 12 preparations after 32 presentations of sound exposure.

In the preparation presented in Figure 11, only one sound exposure was made. The resultant change in position was $10 \mu \mathrm{m}$ and lasted for $30 \mathrm{~min}$, after which a slow return to the original position took place over $90 \mathrm{~min}$. The CM response was reduced and did not recover completely during the time of observation. The sole effect on structure was a reversible sideways swing of the OHCs toward the tunnel of Corti.

Another example of sensitivity loss is seen in Figure 12. After establishing baseline conditions, the organ was exposed to $100 \mathrm{~dB}$ of sound for $5 \mathrm{~min}$. This was well tolerated by the organ, and there was no detectable effect on internal organ structure. Also, the second and third exposures had no effect. However, when the SPL in the fourth exposure was increased to $112 \mathrm{~dB}$, a $5 \mu \mathrm{m}$ displacement of the lipid granule was seen. It was accompanied by a substantial shortening of the OHCs and reduction of the CM, which did not reverse during the time of observation. Because of the strong effect on the electrophysiological response, the sound exposure was prematurely interrupted. Despite this significant effect, structural changes were seen, as described above (Fig. 8). This behavior was encountered in 10 preparations. Within the time of observation, the response did not recover. 

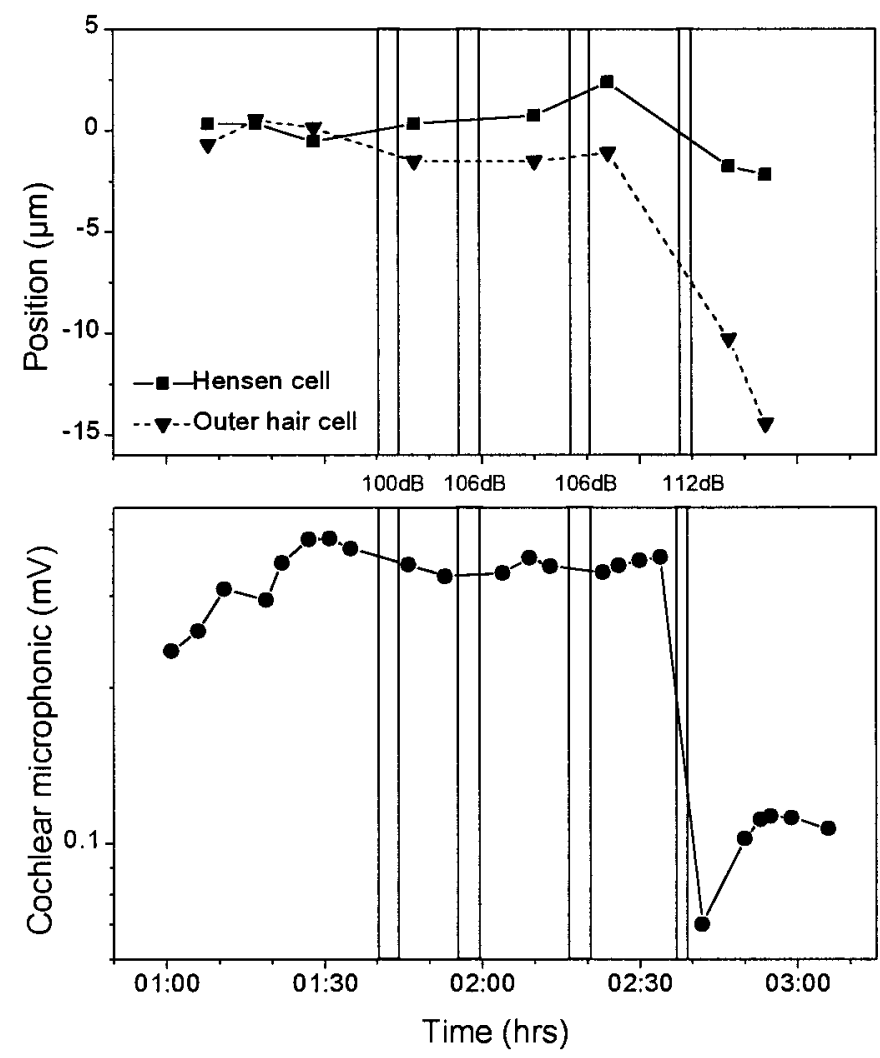

Figure 12. Sustained sensitivity loss. In this organ, there was no noticeable structural change or loss of cochlear microphonic sensitivity after the first three sound exposures. After the fourth exposure, a strong response is seen, with loss of electrophysiological sensitivity, a displacement of the lipid granule, and a shortening of the OHCs (indicated in the top as negative position).

\section{DISCUSSION}

The present report demonstrates two new findings related to acoustic trauma. (1) There are two main and structurally distinct regions inside the organ of Corti in terms of structural conformational changes in response to exposure to intense sound: a stable region around the pillars and IHCs; and a dynamic region around the OHCs, DCs, and HeCs. (2) A dynamic, displacement develops during exposure to intense sound, causing the $\mathrm{HeC}$ complex to swing toward the OHCs. In addition, a loss of physiological sensitivity of the $\mathrm{CM}$ and a number of pathological changes in cell structure were detected after traumatic sound exposures. The findings will be discussed in this order below.

\section{Two regions of structural resilience against acoustic trauma in the organ of Corti}

When time-lapse sequences of events captured before and after sound exposure were viewed, motion and damage were seen to be the least in the region of pillar cells and IHCs and to be the greatest among the OHCs and their surrounding supporting cells. This is in line with previous knowledge about the distribution of noise trauma among the cells in the organ of Corti (Liberman and Dodds, 1984; Kiang et al., 1986). At the end of severe sound exposure, the outer region could be seen contracted with crumpled hair cells and loss of calcein-labeled enzymatic activity, whereas the pillar cells and the IHC retained structure and enzyme activity.

\section{Sound-induced displacement and sensitivity loss}

Dynamic contractions in the hearing organ were seen previously in relation to an increase in intracellular calcium in the OHCs and a drop in the CM (Fridberger et al., 1998). In that study, the organ was viewed looking down at the surface of the sensory organ, and the origin of the motion was not possible to establish. However, with the tilt angle used in the present study, the organ was seen in close-to profile, and the components participating in the motion could be identified. It was found that the contraction consisted of a swinging motion of the DC3s, together with closely related $\mathrm{HeCs}$ (Fig. 2), around a hinge-point generally located between the third row $\mathrm{OHC}$ and DC3. The DCs contain a complex and highly organized cytoskeleton (Slepecky, 1996). A calcium-induced flexion of the phalangeal process of isolated DCs has been demonstrated by Dulon et al. (1994) and could be responsible for the observed motion. They speculate that the DCs may participate in a long-term regulation of cochlear micromechanics during intense sound stimulation.

Two types of shortening or lengthening responses of the OHCs were seen: (1) a reversible contraction or lengthening, sometimes coupled to a tilt toward the tunnel of Corti, resulting in slender undamaged hair cell bodies, or (2) a shortening coupled to a swelling of the cell bodies, followed by blebbing of the apical membrane and loss of enzyme activity. We also observed a swelling of the phalangeal processes of the DCs.

How is the displacement coupled to physiological sensitivity loss? We regard the displacement as a protective response of the organ of Corti to acoustic assault that could lead to injury. It is coupled to a loss of sensitivity of the organ to the test tone. If the intensity or the duration of the acoustic overexposure exceed the protective capacity of the hearing organ, sustained and possibly permanent damage to the OHCs may follow. In both instances, it is possible that calcium ions are involved, as proposed in previous work (Fridberger and Ulfendahl, 1996; Fridberger et al., 1998), perhaps in relation to a depolarization of the hair cells (Cody and Russell, 1985). These are the effects observed on the CM in our preparation, and it is suggested that they contribute, at the cellular level, to the temporary threshold shift and the permanent threshold shift seen after acoustic trauma. We further suggest that the reversible displacement or tilt of the outer region of the organ of Corti is caused by a contractile or disassembly-assembly mechanism in the apical part of the cytoskeleton in the DCs. The temporary sensitivity shift may reflect a protective mechanism, supplied by these cells, which uncouples the more vulnerable parts of the system from excessive mechanical load.

\section{CONCLUSION}

Two types of structural and functional responses were seen to high-intensity sound exposure. The first is a contraction of the DC and $\mathrm{HeC}$ region without any signs of damage to the organ of Corti. This was accompanied by a reduction in electrophysiological responses. The second is a contraction of the $\mathrm{DC}$ and $\mathrm{HeC}$ complex, without any signs of remaining damage to the IHCs but with pathological changes in the OHCs. This was accompanied by a reduction in electrophysiological responses.

We conclude that (1) the supporting cells around the OHCs in the organ of Corti contribute to a protective control mechanism against acoustic trauma; (2) the contraction is coupled to a sensitivity loss; and (3) when the protective capacity of the organ of Corti is overpowered, a sensitivity loss develops as a result of damage to the OHCs. We suggest that these events may be related to the development of hearing loss in vivo. 


\section{REFERENCES}

Borg E, Canlon B, Engström B (1995) Noise induced hearing loss: literature review and experiments in rabbits. Scand Audiol 24:1-147.

Brundin L, Flock B, Flock $\AA$ (1992) Sound induced displacement responses of the guinea pig hearing organ and its relation to the cochlear potentials. Hear Res 58:175-184.

Canlon B (1987) Acoustic overstimulation alters the morphology of the membrana tectoria. Hear Res 30:127-134.

Cody AR, Russell IJ (1985) OHCs in the mammalian cochlea and noiseinduced hearing loss. Nature 315:662-665.

Dulon D, Blanchet C, Laffon E (1994) Photo-released intracellular $\mathrm{Ca}^{2+}$ evokes reversible mechanical responses in supporting cells of the guinea pig organ of Corti. Biochem Biophys Res Commun 3:1263-1269.

Engström B, Flock A, Borg E (1983) Ultrastructural studies of stereocilia in noise exposed rabbits. Hear Res 12:251-264.

Eybalin M (1993) Neurotransmitters and neuromodulators in the mammalian cochlea. Physiol Rev 73:309-373.

Flock Å, Flock B, Fridberger A, Jäger W (1997) Methods for integrating fluorometry in the study of hearing organ structure and function. Hear Res 106:29-38.

Flock Å, Scarfone E, Ulfendahl M (1998a) Vital staining of the hearing organ: visualization of cellular structure with confocal microscopy. Neuroscience 83:215-228.

Flock $\AA$, Flock B, Scarfone E (1998b) Laser scanning confocal microscopy of the hearing organ: fluorochrome-dependent cellular damage is seen after over-exposure. J Neurocytol 27:507-516.

Fridberger A, Ulfendahl M (1996) Acute mechanical overstimulation of isolated outer hair cells causes changes in intracellular calcium levels without shape changes. Acta Otolaryngol (Stockh) 116:17-24.

Fridberger A, Flock A, Ulfendahl M, Flock B (1998) Acoustic overstimulation increases outer hair cell $\mathrm{Ca}^{2+}$ concentrations and causes dynamic contractions of the hearing organ. Proc Natl Acad Sci USA 95:7127-7132.
Kiang NYS, Liberman MC, Sewell WF, Guinan JJ (1986) Single unit clues to cochlear mechanisms. In: Cellular mechanisms in hearing (Flock Å, Wersäll J, eds), pp 171-182. Amsterdam: Elsevier.

Liberman MC, Dodds LW (1984) Single neuron labeling and chronic cochlea pathology. III. Stereocilia damage and alterations of threshold tuning curves. Hear Res 16:55-74.

Lim D (1976) Ultrastructural cochlear changes following acoustic hyperstimulation and ototoxicity. Ann Otol Rhinol Laryngol 85:740-751.

Pickles JO, Osborn MP, Comis SD (1987) Vulnerability of tip links between stereocilia to acoustic trauma in the guinea pig. Hear Res 25:173-187.

Raphael Y, Athey BD, Wang Y, Hawkins JE (1993) Structure of the reticular lamina and repair after noise injury. Rev Laryngol Otol Rhinol (Bord) 114:171-175.

Saunders JC, Cohen YE, Szymko YM (1991) The structural and functional consequences of acoustic injury in the cochlea and peripheral auditory system: a five year update. J Acoust Soc Am 90:136-146.

Slepecky NB (1996) Structure of the mammalian cochlea. In: The cochlea (Dallos P, Popper AN, Fay RR, eds), pp 64-129. New York: Springer.

Spoendlin H (1971) Primary structural changes in the organ of Corti after acoustical overstimulation. Acta Otolaryngol (Stockh) 71:166-176.

Ulfendahl M, Flock $\AA$ (1998) In vitro studies of cochlear excitation. Curr Opin Neurobiol 8:475-479.

Ulfendahl, M, Flock Å, Khanna S (1989) A temporal bone preparation for the study of cochlear micromechanics at the cellular level. Hear Res 40:55-64.

Ulfendahl M, Khanna S, Löfstrand P (1993) Changes in the mechanical tuning characteristics of the hearing organ following acoustic overstimulation. Eur J Neurosci 5:713-723. 